\title{
A Roll Controlling Approach for a Simple Dual-Actuated Flapping Aerial Vehicle Model
}

\author{
Omar El-Farouk E. Labib, Sarah W. El-Safty, Mohamed H. El-Kouly, Mohamed M. Ramadan, Omar M. Shehata, \\ ElSayed I. Morgan
}

Robotics and Autonomous Systems (RAS) Research Group, German University in Cairo (GUC), 5th settlement - New Cairo, 11432 , Cairo, Egypt

\begin{abstract}
Aerial vehicles have been investigated recently in different contexts, due to their high potential of utilization in multiple application areas. Different mechanisms can be used for aerial vehicles actuation, such as the rotating multi-blade systems (Multi-Copters) and more recently flapping wings. Flapping wing robots have attracted much attention from researchers in recent years. In this study, a simple dual-actuated flapping mechanism is proposed for actuating a flapping wing robot. The mechanism is designed, simulated and validated in both simulation and experiments. A roll controlling approach is proposed to control the roll angle of the robot via controlling the speeds of both motors actuating each of the wings. The results achieved are validated experimentally, and are promising opening the door for further investigation using our proposed system
\end{abstract}

\section{Introduction}

In recent decades, Unmanned Aerial Vehicles (UAVs) has encountered a consistent ascent, specifically Micro Air Vehicles (MAVs) hold a unique sense of interest among engineers for both domestic and military applications. MAVs that fly utilizing flapping wings hold great potential for in- door surveillance and outdoor applications like photography and exploration. Progresses in the comprehension of flapping wing vehicles and flapping wing aerodynamic properties have incited a few endeavors to create flapping flight vehicles.

Endeavors to date are principally concerned with the attain- ability of these vehicles, as opposed to the advancement of their execution or the foundation of general outline stan- dards. Examinations have concentrated on amplifying thrust to-weight or minimizing power consumption. Work in the outline and advancement of individual vehicle subsystems incorporate endeavors to enhance stroke kinematics, wing shape and consistence, transmission proficiency and actuator execution.

The rest of this paper is organized as follows, section 2 presents an overview about research endeavors conducted by other researchers tackling the same problem. In section 3, the design and fabrication details are conducted, while section 4 presents the control and simulations conducted. Results are presented in section 5, and finally section 6 concludes this paper..

\section{Literature review}

In this section, investigation of related research efforts is conducted. This section is divided into two parts, The first will cover different designs investigated by researchers, and the second will be on the various control studies conducted.

\subsection{Design review}

To mimic the flapping wing movement seen in nature, particularly at small scales, MAVs have used just a few types of actuation effectively; piezoelectric and electromag- netic actuation. These sorts of actuation when combined with mechanical transmission subsystems, most popular being the ranges between 6-bar and 4-bar mechanisms [1] can produce improved stroke amplitudes and responding motion needed for flapping flight [2].

While the single actuator and wing is able to produce lift capa- ble of overcoming its own weight, this is only viable when the actuator is mounted rigidly to a fixture. To achieve free flight, the mean and instantaneous body forces and torques, or body wrench, delivered by a single flapping wing would prompt both lessened flapping sufficiency and unstable spinning. To make a powerful free-flight, extra actuators and wings must be added to counter this instantaneous torque [3]

In [4], a small butterfly-style flapping robots with wing veins and investigated their flight characteristics for different design parameters such as swept-forward wing angle and center of mass (COM). The experimental results showed that the body pitch angle was controlled 
by the swept-forward wing angle and by the relative positions of the COM and center of lift (COL).

In [5] the design and construction of a 2.6 gram electromag- netic actuator operated at resonance is presented. Independent bench tests for the coil and spring magnets were used to modify analytical models of the actuator, derived in detail in a parallel study. Wing kinematics and mean lift measurements were performed for the flapper operating at resonance, producing a lift-toweight ratio for the actuator of over one at $24 \mathrm{~V}$.

In this study, it is desired to achieve a design that utilize 2 actuators to drive a mirrored mechanism, in order to be able to control each motor separately. This would be later used to experiment with the effects of variation in wing speeds on roll, using a developed test platform to isolate that degree of freedom which will be explained in details in the upcoming sections.

\subsection{Control review}

Several researchers investigated the problem of control of aerial vehicles. In [6] researchers have tried to investigate the potential of four design alternatives based on a piezoelectric driven passive pitch reversal wing. The models are compared based on their performance under an ideal linear quadratic reg- ulator (LQR) control scheme. The developed designs showed promising feasible controllable vehicles results.

While in [1], Liu, et al investgated the flight stability of a prototype flapping aerial vehicle. An integrated study of flexible wing aerodynamics and passive dynamic flight stability of the MAV by a combination of flexible wing kinematics and force measurements and computational approaches were conducted and simulated.

In this study, continuation upon previous researchers efforts is conducted in order to design and control a dualactuated flapping wing robot, and control its roll-angle.

\section{Mechanical design and fabrication}

In this section, we will discus the process of reaching the final design to achieve our desired outcome, which will be explained in details in the coming subsections. The design iterations will be explained, the drawbacks and advantages of each design, and the fabrication process.

\subsection{Designing Process}

We stared initially using 2 servo motors, each connected to a wooden link. Both where fixed together using a wooden disc and suspended by a rod to view the effects on roll. The servo motors were slow and required us to change the direction of rotation to flap which was not efficient.

We decided to change the actuators to dc motors, and imple- ment a motion converting mechanism. In our first iteration of this idea, we developed a 6-bar mechanism. The system was manufactured using acrylic which is a commercial and light- weight material suitable for the flapping wing mechanism. The problem with the 6-bar mechanism was that it was too heavy and inefficient, The height of the mechanism was $26 \mathrm{~cm}$.

A 4-bar mechanism was then designed, which greatly increased the compactness of the system. The final dimensions of the mechanism without the motors was 4.5 $\mathrm{cm}$ high, $9 \mathrm{~cm}$ wide and $3 \mathrm{~mm}$ thick. We mirrored the mechanism and made a full assembly with the $2 \mathrm{dc}$ motors mounted on the final design. The final measurements of the mechanism without the motors was $4.5 \mathrm{~cm}$ in height, $9 \mathrm{~cm}$ width, and a thickness of $3 \mathrm{~mm}$.

\subsection{Design Analysis}

Analysis of the mechanism was done twice; once without wings attached to the flapping links, and another using wings that we designed and will be disused in the wing design section. Tests were conducted on the wingless model, the wingless mechanism would act as a reference for testing different wing designs later if needed.

In the wingless tests, we first analyzed our mechanism experimentally using video tracking software called (Tracker) by (OSP) Open source physics. Track points were added to the tip of each flapping link, to be able to track its displacement in both $\mathrm{X}$ and $\mathrm{Y}$ axis, velocity components and magnitudes, and finally acceleration components and magnitudes. We tracked the mechanism starting from an operational voltage of 2 volts till we reached 12 volts, which is shown in the following graphs.

Mathematical analysis of the mechanism was done using (Matlab), the equations of the 4-bar mechanism were plotted using matlab and then compared with the video tracking software's results. The parameters of our mechanism are all sown in the following table.

\begin{tabular}{|l||l|}
\hline \multicolumn{2}{|c|}{ Mechanism Parameters } \\
\hline Parameter & Symbol \\
\hline Ring radius & $l_{1}$ \\
Connector & $l_{2}$ \\
Flapper extension & $l_{3}$ \\
Hinges Distance & $l_{4}$ \\
Motor position & $\phi$ \\
Flapping angle & $\theta$ \\
Center of mass X & $P_{x}$ \\
component & \\
Center of mass Y & $P_{y}$ \\
component & \\
\hline
\end{tabular}

$$
\begin{gathered}
k_{1}=l_{4} / l_{1} \\
k_{2}=l_{4} / l_{3} \\
k_{3}=\frac{l_{1}^{2}-l_{2}^{2}+l_{3}^{2}+l_{4}^{2}}{2 l_{1} l_{3}} \\
A=\cos \phi \quad-k 1 \quad-k 2 \cos \phi+k 3 \\
B=-2 \sin \phi \\
C=k_{1} \quad-\left(k_{2}+1\right) \cos \phi+k_{3} \\
\theta=2 \arctan \frac{-B-\sqrt{B^{2}-4 A C}}{2 A}
\end{gathered}
$$




\subsection{Wing design and fabrication}

To be able to test the effect of a flapping wing on our mechanism, we went for a minimalistic design. The reason being that we are investigating the effects of lift and thrust forces that are added to the system by the wings, and how they affect the roll. Takeoff with the current design is not the goal of this model, we are only using it as a testing platform to base our controller that will be discussed in details in the control and simulation section.

A symmetric wing was designed using using Computer aided design software (SolidWorks). The skeleton and connector are made up of acrylic. The skeleton is $25 \mathrm{~cm}$ long, $15 \mathrm{~cm}$ wide and $3 \mathrm{~mm}$ thick. The wings were made up of stencil paper as it is thin and durable.

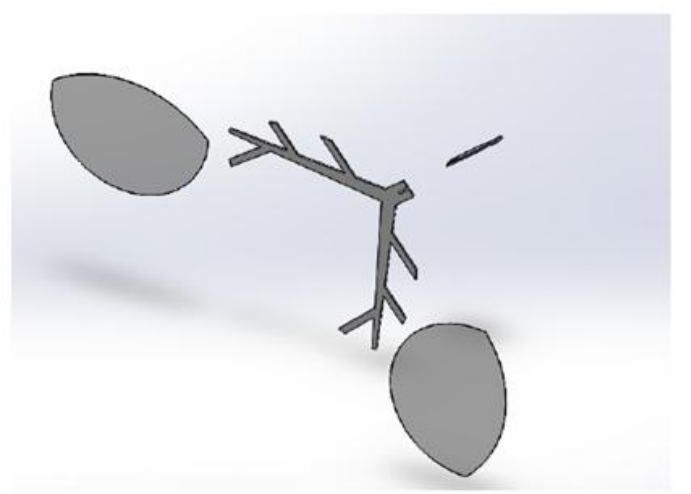

Figure 1. Wing assembly

\subsection{Test bed}

Finally a test bed was made to be able to run various test scenarios while implementing our controller model. The test bed was a simple design, a housing of dimensions 80 $\mathrm{cm}$ in height, $65 \mathrm{~cm}$ in length and width. Suspended in its interior are 2 supports to be connected to a rod that isolates roll only. The mechanism is given space and clearance to fully rotate at 360 degrees. as shown in Fig. 2.

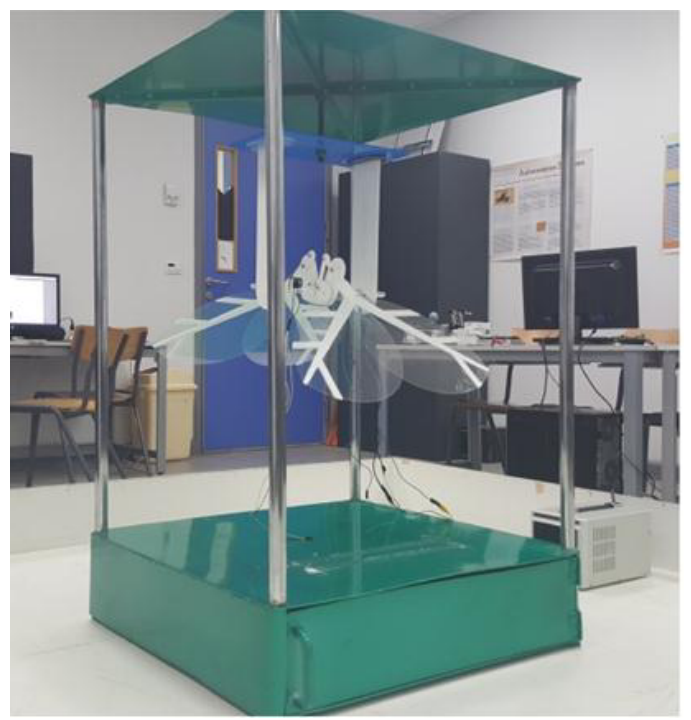

Figure 2. The testing platform : Bed and mechanism

\section{Controllability analysis}

The two DC-motors produce one effect that alter the forces and moments produced by wing motion. When the wing angle is changed, the resulting wing motion function remains a periodic function as shown in the design section regardless the speed of the motors. The difference is the frequency of which the moment arms of the wings change, hence, moments are altered. This effect can either be manipulated to change the moments of the whole system thus controlling roll as wanted, or manipulated to counteract external forces such as the counter torque mechanism of a helicopter.

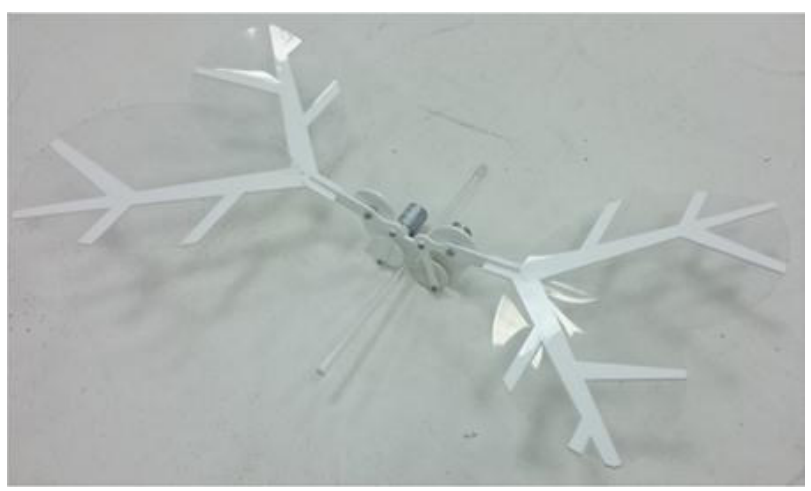

Figure 3. The dual actuated flapping wing mechanism

\subsection{Simulations}

In the coming sub-sections, the three scenarios chosen for theory validation will be demonstrated. The first is stabilizing the model at a reference roll angle, while acted upon by external forces. The second is tilling the model to a desired angle. The third scenario utilizes the capabilities of our ap- proach, as we run a simulation of a predetermined sequence of commands. Simmechanics and Simulink were used to run simulations of the different scenarios. This was also done experimentally but only the second scenario was tested. The control of our model is primarily dependent on changing the DCmotor speeds while obtaining feedback from both the encoders and an inertial measurement unit (IMU).

\subsection{Stabilization scenario}

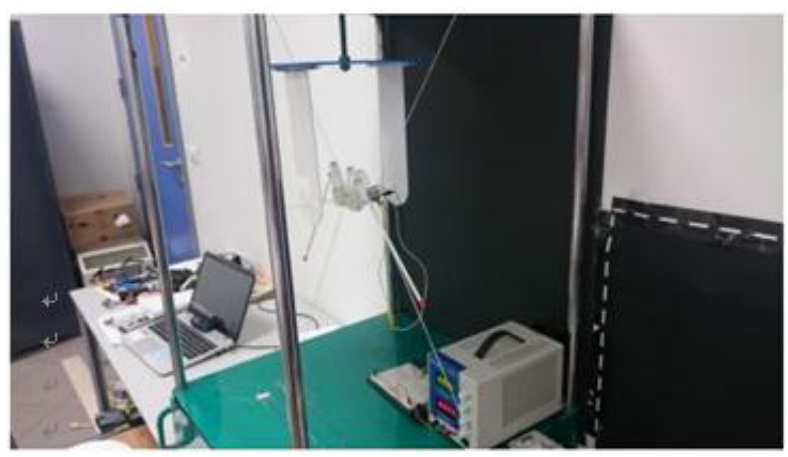

Figure 4. The first test scenario conducted

The stabilization scenario was conducted by adding a constant external force that would change the roll of the 


\section{ICIEA 2016}

model. By using feedback from the roll angle, the angular velocity of each motor is changed to compensate this disruption. A PID controller is used for this closed loop, two scopes for the rpm of each motor separately and one scope for the roll of the model as can be seen in Fig. XX.

\subsection{Desired angle scenario}

In this scenario, a desired angle is given as an input and the speed of each motor is separately regulated using PID control till the desired angle is achieved by the model.

\subsection{Predetermined sequence}

The final scenario was a predetermined sequence of commands; stabilizing at reference angle 1 for 3 seconds then reference 2 for 2 seconds then back to reference 1 again indefinitely.

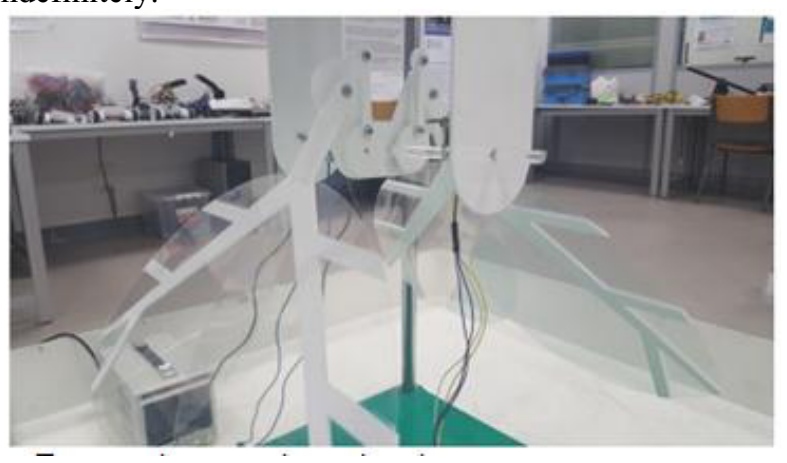

Figure 5. The second test scenario conducted

\section{Results}

The final design in our experimental modeling platform was able to produce sufficient moments and torques to change its roll efficiently and reliably. Three test scenarios were done, the first being the stabilization scenario which responded as expected giving satisfactory results but with some oscillations which can be overcome using different controllers such as fuzzy controller or tuning the PID values to minimize the overshooting. The second test scenario gave similar results but with less oscillations as there were no external forces acting upon the system. The third scenario obeyed the sequence given but the settling time was too long but can be refined by tuning the PID values.
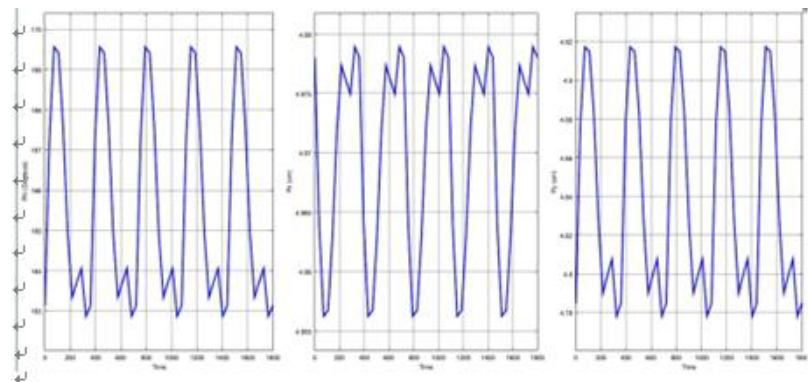

Figure 6. Phi, Px, and Py versus time

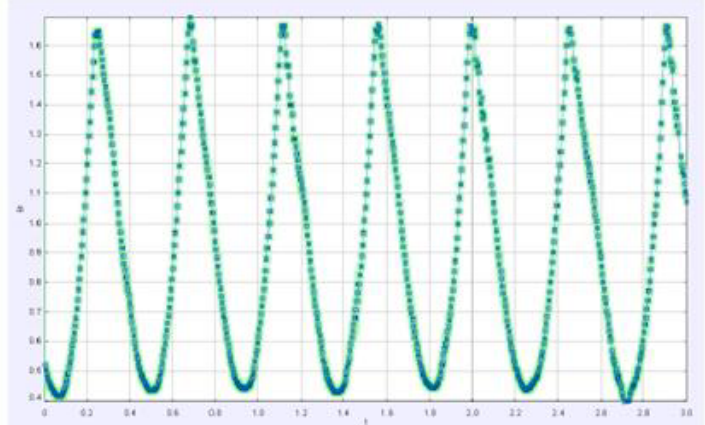

Figure 7. Angle at 8 volts

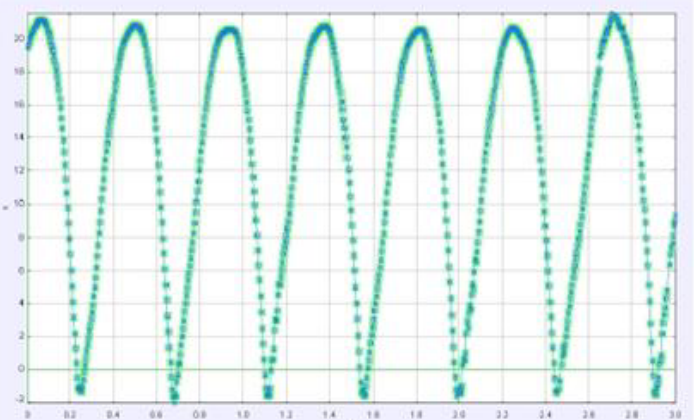

Figure 8. $\mathrm{X}$ position at 8 volts

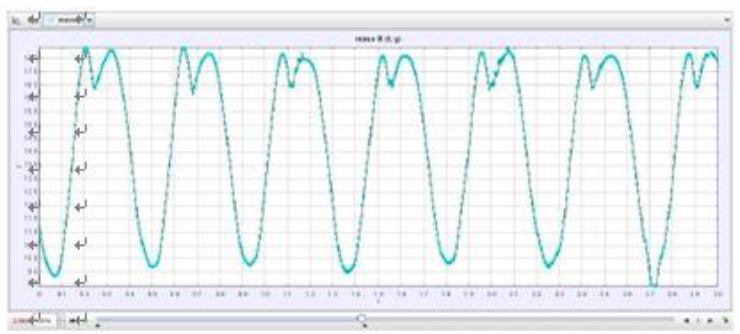

Figure 9. Y position at 8 volts

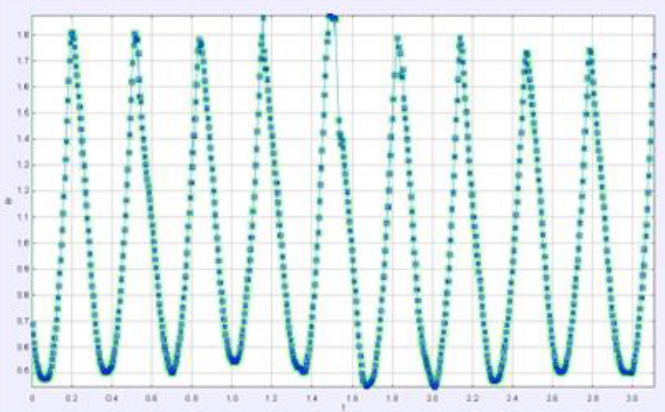

Figure 10. Angle at 12 volts

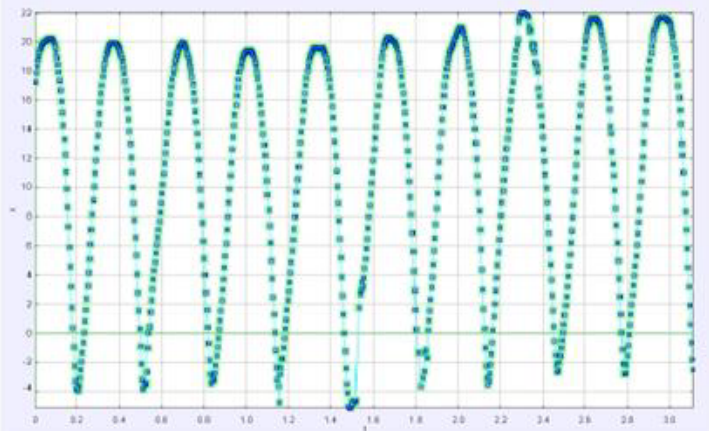

Figure 11. $\mathrm{X}$ position at 12 volts 


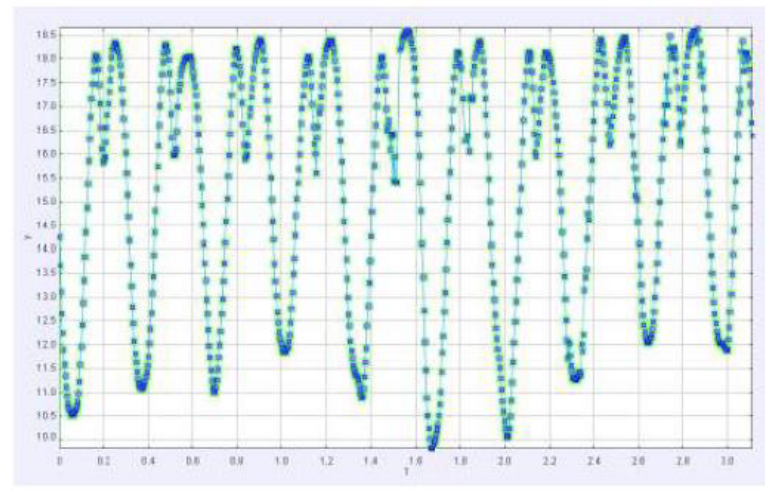

Figure 12. Y position at 12 volts

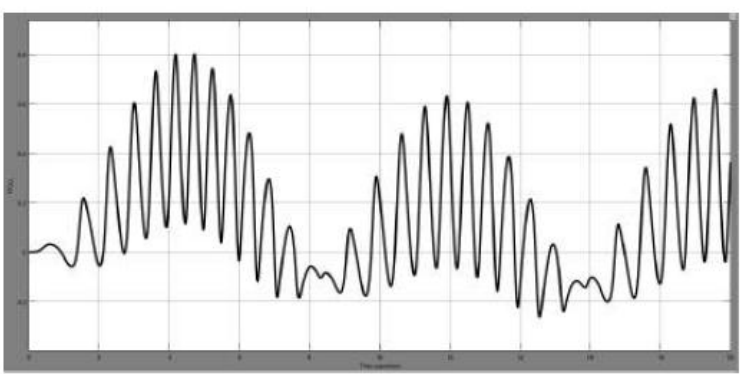

Figure 13. Scenario 1 Roll

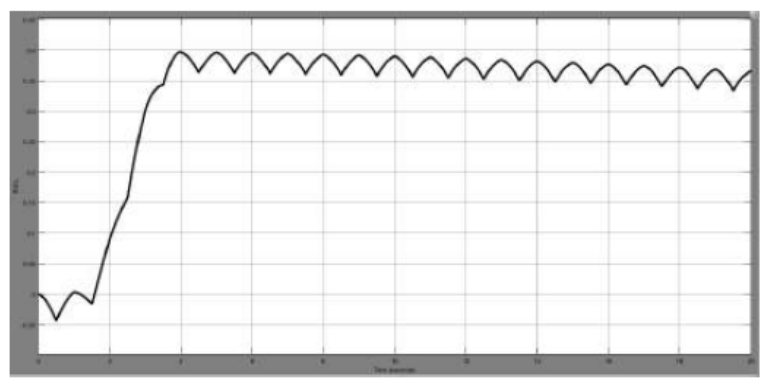

Figure 14. Scenario 2 Roll

\section{Limitations}

Many limitations were faced in the study; there was no variety in the commercially available components or materials. Moreover, the availability of DC motors with built-in encoders was limited. Also, the shaft encoders available were not compatible with the motors used.

\section{Conclusion and future work}

In this study we were aiming to tackle the stability problems which lead to short flight times of flapping wing MAVs. Using two actuators, one for each wing instead of a single one opens a lot of possibilities for the future of flapping flight. We designed a dual-actuated flapping MAV model which can be used as a testing platform for different roll control approaches. Which will help to build a better understanding of how to control this degree of freedom, and hopefully result in more stable flapping air vehicles and thus longer and more reliable ones. Our design was not aiming for take-off at this stage; however, due to the importance of roll in achieving longer flight times, the model is chosen to be as simple and general as possible to be easily tested.

For future work, using this platform to test various controllers and there effect on the system. Adding pitch control to the platform by using a method to manipulate it such as Festo's Smart bird's neck and tail actuation. Developing a smaller testing platform capable of takeoff using the same feedback method to further analyze the behavior.

\section{Acknowledgements}

The Authors would like to thank Professor Yasser Higazi Dean of the Information and Electronics Technology (IET)and Professor ElSayed Imam Morgan Dean of Mechatronics Engi- neering for there support and efforts to get these results out. It wouldn't have happened without you, thanks.

\section{References}

1. H. Liu, X. Wang, T. Nakata, and K. Yoshida, Aerodynamics and flight stability of a prototype flapping micro air vehicle, Complex Medical Engineering (CME), 2012 ICME International Conference on. IEEE, pp. 657-662, (2012)

2. T. Zhang, C. Zhou, and S. Su, Design and development of bio-inspired flapping wing aerial vehicles, Advanced Robotics and Intelligent Systems (ARIS), 2015 International Conference on. IEEE, pp. 1-6, (2015)

3. L. Hines, D. Colmenares, and M. Sitti, Platform design and tethered flight of a motor-driven flappingwing system, in Robotics and Automation (ICRA), 2015 IEEE International Conference on. IEEE, pp. 5838-5845, (2015)

4. T. Fujikawa, Y. Sato, Y. Makata, T. Yamashita, and K. Kikuchi, Motion analysis of butterfly-style flapping robot for different wing and body design, Robotics and Biomimetics, 2008. ROBIO 2008. IEEE Inter- national Conference on. IEEE, pp. 216-221, (2009).

5. J. Roll, B. Cheng, X. Deng, et al., Design, fabrication, and experiments of an electromagnetic actuator for flapping wing micro air vehicles, Robotics and Automation (ICRA), 2013 IEEE International Conference on. IEEE, pp. 809-815, (2013)

6. L. L. Hines, V. Arabagi, and M. Sitti, Control performance simulation in the design of a flapping wing micro-aerial vehicle, Intelligent Robots and Systems (IROS), 2010 IEEE/RSJ International Conference on. IEEE, pp. 1090-1095, (2010) 